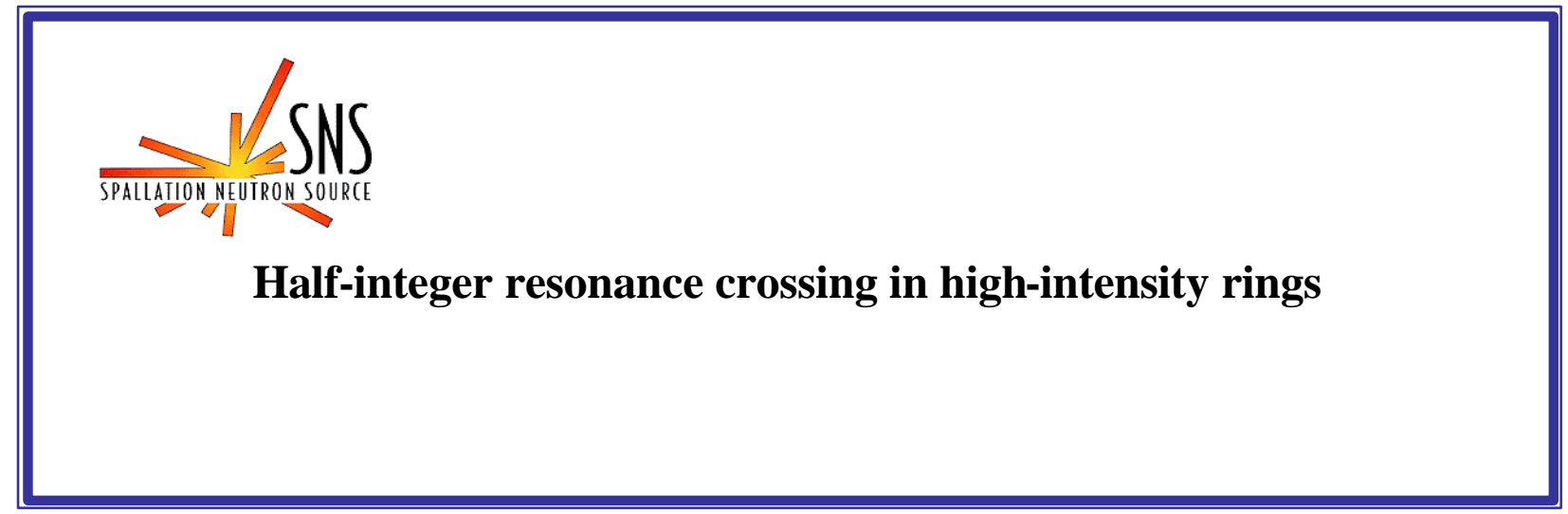

BNL/SNS TECHNICAL NOTE

NO. 100

A.V. Fedotov and I. Hofmann

September 26, 2001

COLLIDER-ACCELERATOR DEPARTMENT

BROOKHAVEN NATIONAL LABORATORY

UPTON, NEW YORK 11973 


\title{
Half-integer resonance crossing in high-intensity rings
}

\author{
A.V. Fedotov and I. Hofmann \\ CA Department, Brookhaven National Laboratory, Upton, New York 11973; \\ GSI Darmstadt, Planckstr 1, 64291 Darmstadt, Germany
}

(September 26, 2001)

\begin{abstract}
A detailed study of the influence of space charge on crossing second order resonances is presented and associated with the space-charge limit of highintensity rings. Two-dimensional (2D) simulation studies are compared with envelope models, which agree in the finding of an increased intensity limit due to the coherent frequency shift. This result is also found for realistic bunched beams with multi-turn injection painting. Characteristic features like the influence of tune splitting, of structure resonances and the role of envelope instabilities are discussed in detail. The theoretical limits are found in good agreement with the performance of high intensity proton machines.
\end{abstract}

\section{INTRODUCTION}

The importance of space charge was realized at an early stage of design of high-intensity rings. A figure of merit became a space-charge induced shift of individual-particle betatron tunes. In conjunction with single-particle framework of betatron resonances this resulted in a space-charge limit argument for circular accelerators. However, such a widely used criterion, which is based on the resonance condition for incoherent tunes, overestimates the threshold for onset of resonances, and, what is equally important, incorrectly describes the 
resonance response. A correct treatment requires one to take into account the collective behavior of the beam. Understanding such collective beam behavior near the resonances as well as associated resonance crossing is of crucial importance for the next generation high-intensity rings under construction and design. It also provides the framework for the intensity upgrade of existing high-intensity machines.

In this paper we demonstrate the validity of collective beam response to betatron resonances using an example of the Spallation Neutron Source (SNS) accumulator ring which is currently under construction [1]. We also describe the main features of beam envelope resonant response and its application to the space-charge limit in the high-intensity rings. We first confirm this collective resonance theory for a two-dimensional unbunched beam with various beam distributions. We then extend our studies to a realistic bunched beam as well as the process of beam accumulation by multi-turn injection. As a result, we explore the applicability of collective-resonance theory to a realistic beam in high-intensity circular accelerators.

The structure of the paper is as follows. Section II is devoted to an overview of coherent resonance theory with an application to the space-charge limit in a ring. Section III demonstrates application of this theory with regard to a half-integer resonance driven by the gradient errors. In this section both the space-charge limit and envelope response issues are discussed. Section IV compares beam envelope response to an imperfection resonance with a similar response to a structure resonance. In particular, one of the proposed SNS working points is examined and the resulting space-charge limit is discussed. Section $\mathrm{V}$ is devoted to a discussion of the coherent resonance condition and its application to the envelope instability. Finally, Section VI summarizes the major points which follow from the coherent-resonance response. 


\section{SPACE-CHARGE LIMIT}

\section{A. Coherent resonance theory}

In the absence of coupling, the resonance condition for the tune can be written as $n / m=$ $\nu_{0}$, where $n$ is the harmonic content of the errors and $m$ is the resonance order. The resonance order $m$ can be associated with the multipole spectrum of the lattice errors, with $m=2$ corresponding to gradient errors, $m=3$ to sextupole errors, etc. If we now think about space charge as a perturbation producing a tune shift of individual particles (incoherent tune shift) the resonance condition would become $n / m=\nu_{i n c} \equiv \nu_{0}-\Delta \nu_{s c}$, with $\Delta \nu_{s c}$ being the maximum space-charge tune shift. Such a criterion is widely used when one wants to choose the best working point in the tune space by avoiding dangerous resonances. However, this condition, based on a single-particle framework, cannot correctly describe the onset of the resonance and provide the proper picture of beam-envelope resonant response. For high-intensity accelerators this condition gives too conservative an estimate for loworder resonances which are most important in consideration of the resonance condition and underestimates the maximum achievable current. A correct treatment requires one to take into account the collective behavior of the beam.

The fact that the incoherent tune is inadequate to describe integer resonances was first emphasized by Morin [2] and Lapostolle [3]. It was then Smith [4] who used the envelope equation to prove that the half-integer resonance does not occur at the incoherent frequencies either. Smith's analysis was extended to high-order resonances by Sacherer [5] using the onedimensional (1-D) Vlasov equation. The theoretical framework was later extended to twodimensions (2-D) by Gluckstern [6]. This theory was subsequently confirmed with computer simulations by Hofmann [7] and Machida [8]. Recently, a very useful overview was presented by Baartman [9].

The incoherent space-charge approach to the second order resonance condition fails because it is based on the assumption that the beam size remains constant. However, the 
beam envelope depends on the oscillation amplitude of the individual particles. Thus, if the gradient error causes these amplitudes to grow, the beam size also grows which in turn reduces the space-charge effect (this, of course, applies to high-order multipole errors also). Clearly, the incoherent space-charge approach is not self-consistent. More than that, using the KV beam, it is easy to show [10], [5] that the effect of gradient errors in the lattice is exactly compensated by the space-charge perturbation induced by those errors if $\nu_{\text {inc }}=n / 2$. Similar result can be obtained for high-order resonances using the Vlasov equation [11].

In general, the coherent resonance condition has the form:

$$
n=\Omega_{m} \equiv m \nu_{0}-\Delta \Omega_{m},
$$

where $\Omega_{m}$ is the frequency of the mth-order coherent beam mode, and $\Delta \Omega_{m}$ is the coherent space-charge tune shift of the mth-order mode from its zero-current value $\left(m \nu_{0}\right)$. In principle, an external driving potential in the form $x^{k} \cos \left(m \nu_{0} \theta\right)$ can drive collective beam modes with $k \neq m$. It has been suggested that modes with $k \neq m$ should not be expected to play a significant role for beams with realistic non-KV distributions [9]. The resonance condition of Eq. 1 takes into consideration that the resonance is coherent in nature and eliminates a possible confusion with the incoherent resonance condition. However, for practical estimates of the space-charge limit in a ring it is not very illuminating since the allowed maximum intensity is typically calculated through the maximum space-charge tune shift of individual particles. Therefore, we follow the notation of Ref. [9] and express the coherent space-charge tune shift as $\Delta \Omega_{m}=m C_{m} \Delta \nu_{s c}$. The coherent mode frequencies for any order of $m$ were derived both for the axi-symmetric [6] and non axi-symmetric [12] beams. The corresponding coefficients $C_{m}$ can be easily extracted from Refs. [6], [11], [12] and are summarized for example in Ref. [9].

In this paper we consider only the $m=2$ case which is associated with the space-charge limit imposed by the half-integer resonance. For a two-dimensional round beam $(a=b)$ there are two coherent modes of beam envelope oscillation. For the case of very close zero-current tunes, $\left|\nu_{0 x}-\nu_{0 y}\right| \ll \Delta \nu_{s c} / 4$, one refers to these modes as symmetric ("in-phase") mode 
with $\Delta \Omega_{2, s y m}=\Delta \nu_{s c}$ and antisymmetric ("out-of-phase") mode with $\Delta \Omega_{2, a s y m}=\frac{3}{2} \Delta \nu_{s c}$. As a result, the space-charge limit (coherent-resonance condition for the $n / 2$ resonance) is expected around beam intensities $\Delta \nu_{s c} / C_{2}$, which is $2 \Delta \nu_{s c}$ and $\frac{4}{3} \Delta \nu_{s c}$ for the symmetric and antisymmetric modes, respectively. Note that if $a \neq b$, the coefficient $C_{2}$ depends on the transverse beam sizes $a$ and $b$ [5], [9]. For the split-tune case, $\left|\nu_{0 x}-\nu_{0 y}\right| \gg \Delta \nu_{s c} / 4$, the two envelope modes are essentially decoupled. Although the in-phase and out-of-phase symmetry of the mode oscillations is still preserved, one can regard such oscillations as onedimensional with approximately the same coherent space-charge tune shift in both transverse planes: $\Delta \Omega_{2, s p l i t}=\frac{5}{4} \Delta \nu_{s c}$. The resulting coherent space-charge limit is then $\frac{8}{5} \Delta \nu_{s c}$.

\section{B. Non-uniform distributions}

The collective beam modes and coherent resonance condition were derived using the uniform-density KV beam. However, it was shown by Sacherer [22] that one can use rms envelope equation for non-KV distributions as well, using the rms quantities. This allows us to use second-order coherent modes for non-KV beams and treat the coherent envelope response to the half-integer resonance using the rms envelope equation. This concept of KV equivalent beams has been used in studies of high-order resonances as well [23], [9], [8].

It turns out, that for a non-uniform distribution the difference between a single-particle approach and coherent resonance condition becomes even more pronounced. This important feature was specifically emphasized by Machida [8] and Baartman [9]. A significant difference is due to the fact that the coherent resonance condition, given by Eq. 1, when applied to non-uniform beams assumes the rms equivalent uniform beam (KV) tune shift. However, for non-linear distributions the maximum space-charge tune shift is bigger than the one of a uniform beam. For example, for a Waterbag (WB) distribution $\Delta \nu_{s c, \max }=1.33 \Delta \nu_{K V}$, and for a Gaussian distribution $\Delta \nu_{s c, \max }=2 \Delta \nu_{K V}$. As a result, the incoherent space-charge tune shift can significantly exceed a single-particle resonance condition until the coherent resonance condition for an rms equivalent $\mathrm{KV}$ beam is met. 


\section{Decoherence effects}

The theory of collective beam-envelope response to a resonance at coherent frequencies is based on a two-dimensional beam model. For bunched beams the onset of the resonance can be predicted to some extent by using the bunching factor parameter and thus considering the resonance condition for the portion of the beam in the vicinity of the highest longitudinal charge density. However, to what extent this coherent resonant response is altered in the presence of other effects, such as synchrotron motion, beam energy spread, multi-turn injection, etc., is not obvious without dedicated study. One may expect that impact of such effects is not strong for the SNS beam parameters due to the very slow synchrotron motion. These effects are explored in Section IV with an application to the SNS accumulator ring.

\section{HALF-INTEGER IMPERFECTION RESONANCE}

To demonstrate the coherent-resonance theory we have chosen the SNS working point with zero-current tunes $\left(\nu_{0 x}, \nu_{0 y}\right)=(6.45,4.6)$. The gradient error is then introduced in order to investigate the half-integer resonance with harmonic $n=9$.

\section{A. Coherent space-charge limit}

The single-particle approach predicts a resonance condition at an intensity which corresponds to the space-charge tune shift of $\Delta \nu_{s c}=0.1$ in the vertical direction. For a beam with a uniform density, the tunes of all particles are thus placed at $\nu_{y}=4.5$ when $I \equiv \Delta \nu_{s c} / \Delta \nu_{i n c}=1$. Here, the incoherent space-charge limit $\Delta \nu_{i n c}$ is defined as the distance from the bare tune to the half-integer resonance line: $\Delta \nu_{i n c} \equiv \nu_{0 y}-n / 2$. For non-uniform distributions, the incoherent resonance condition occurs for the intensity parameter $I$ less than unity (if $I=1$ is taken to be an intensity where the resonance condition for a uniformdensity beam is satisfied) because the maximum space-charge tune shift of a non-uniform beam is larger than the one of a uniform-density beam. Specifically, for a Waterbag (WB) 
distribution $\Delta \nu_{s c, \max }=1.33 \Delta \nu_{K V}$, where $\Delta \nu_{K V}$ is the space-charge tune shift of a beam with uniform-density KV distribution. As a result, the incoherent space-charge limit for a WB beam is expected at intensity $I=0.75$. On the other hand, the coherent beam resonance is expected at similar intensities both for a $\mathrm{KV}$ and $\mathrm{WB}$ beam. For the working point discussed in this example, $\left|\nu_{0 x}-\nu_{0 y}\right| \gg \Delta \nu_{s c} / 4$, the coherent resonance condition is expected around $I=8 / 5$. Space-charge limits discussed above are summarized in Fig. 4 which shows the collective beam response to the $n / 2=4.5$ resonance. In this figure, the green vertical line indicates the incoherent space-charge limit for a WB beam, the pink line corresponds to the incoherent space-charge limit of a uniform density beam, while the red line shows the coherent resonance condition.

\section{B. Beam envelope response}

Some important features of space-charge effects on crossing of the half-integer resonance, such as coherent frequency shift, nonlinear detuning and saturation of beam envelope growth, can be retrieved from the envelope equations [5]. Obviously, the effect of frequency spread - as a possible source of decoherence - and the filamentation in the phase space due to halo formation are beyond the level of description of an envelope model. Furthermore, the possibility of envelope instability needs to be considered in some special cases as will be shown in Section V.

Noting that the effect of the error resonance depends primarily on the tune and not the details of the focusing lattice, we start with an assumption of constant focusing. We

consider the half-integer resonance near the unsplit-tune working point $\nu_{0, x, y}=4.6$ and assume $n=9$, where $n$ stands for the error Fourier harmonic. Hence, the expected coherent resonance condition is

$$
\Omega_{2}=2 \nu_{0}-\Delta \Omega_{2}=9
$$

where $\Delta \Omega_{2}$ is the coherent space-charge tune shift of second-order modes $\Omega_{2}$. An antisymmetric error in $x$ and $y$ (as would be produced by a single quadrupole) then drives an 
out-of-phase mode resonance, while a symmetric error is needed to drive the in-phase mode resonance (note that for sufficiently split tunes a single quadrupole error will drive both modes).

We assume equal emittances in $x$ and $y$ and solve the envelope equations with error Fourier harmonics of $1 \cdot 10^{-3}$ units (relative to the unperturbed focusing constant) and a small initial envelope mismatch of $2 \%$. To demonstrate the effect of space charge on the envelope, Fig. 1 shows the case of a symmetric error driving the in-phase mode resonance with beam parameters such that the single-particle tune is just on resonance (incoherent depressed tune $\left.\nu_{x, y}=4.5\right)$. The envelope undergoes a small periodic beating due to the proximity of the coherent tune to the resonance; note the relative smallness of the effect for the incoherent tune sitting exactly on resonance. The maximum envelope excursion grows with increasing beam intensity (decreasing of the depressed tune $\nu_{x, y}$ ), which brings the coherent mode frequency closer to the resonance. In Fig. 2 we show the maximum envelopes for this case as the function of depressed incoherent tune $\nu_{x, y}$ for both the symmetric and anti-symmetric errors, which drive the in-phase and out-of-phase modes, respectively. The envelope increases noticeably only with the coherent frequency crossing the integer, which occurs at $\nu_{x, y}=4.467$ (beam intensity equal to $\frac{4}{3} \Delta \nu_{i n c}$ ) for the out-of-phase mode, and at $\nu_{x, y}=4.4$ (beam intensity equal to $2 \Delta \nu_{i n c}$ ) for the in-phase mode. Due to the nonlinear dependence (increase) of the envelope eigenfrequency with amplitude the maximum growth happens for higher beam intensities at $\nu_{x, y}=4.44$ for the out-of-phase and $\nu_{x, y}=4.37$ for the in-phase mode. For completeness, a zero-current envelope response to this $1 / 2$ resonance is obtained by varying the working point $\nu_{0, x, y}$.

The size of the maximum envelope excursion, as well as the width of beam envelope response curve, is a function of the strength of the imperfection resonance (magnitude of an error). To demonstrate this effect, Fig. 3 shows the maximum $y$-envelopes for the split-tune working point $\left(\nu_{0 x}, \nu_{0 y}\right)=(6.45,4.6)$ and three different magnitudes of an error: $3 \cdot 10^{-3}, 1$. $10^{-3}, 1 / 3 \cdot 10^{-3}$ (an anti-symmetric error was chosen, but the results are very similar for a symmetric error). The intensity parameter $I \equiv \Delta \nu_{s c} / \Delta \nu_{i n c}$ (abscissa) is expressed as 
space-charge tune shift normalized to the distance from the bare tune to the half-integer $\left(\Delta \nu_{i n c}\right)$. Such a response diagram explicitly shows the beneficial effect of the coherent resonance condition compared to the incoherent space-charge limit, corresponding to $I=1$. Note that the coherent envelope frequency is exactly on resonance for the normalized tune shift of 1.635 , the number obtained by direct solution of the full dispersion relation for the coherent frequency. This number is slightly higher than the approximate coefficient $8 / 5$. The stopbands shown in this picture are related to the out-of-phase mode, while the in-phase mode resonance would appear if $\nu_{0, x}$ were chosen correspondingly above 6.5 . The strongly asymmetric shape of the envelope response curves is a result of the nonlinear nature of the envelope equation, in particular the increase of envelope frequency with amplitude. The basic features of such response curves are summarized in Appendix A. We now proceed to the realistic SNS lattice with the working point at $\left(\nu_{0 x}, \nu_{0 y}\right)=(6.45,4.6)$. The gradient error is introduced in a single quadrupole with the normalized strength of an error $\Delta k=2.5 \cdot 10^{-3}$ units. Simulations were done using the Particle-In-Cell (PIC) code ORBIT [20]. The results of simulations are presented in Fig. 4, which confirm the beam envelope response expected based on the envelope equations (Fig. 3). All major features of the coherent resonance response are transparent: resonance happens at higher intensity than the incoherent spacecharge limit, it leads to a finite increase of beam envelopes, and the maximum envelope amplitudes are reached at intensities higher than the middle point of the resonance. Note that our statement about finite envelope excursions during the resonance crossing in the direction of the normalized tune shift (intensity) increase is true only for simulations with a fixed intensity for each individual run. This allows the possibility of getting out of the resonance condition with envelope oscillations around a small-amplitude stable fixed point. With a slow adiabatic increase of beam intensity the beam envelopes are expected to grow with oscillations around large-amplitude stable fixed points (see Appendix A). Both of these features of an envelope response were recently demonstrated using the envelope equations and PIC simulation with a multi-turn injection for the LANL PSR [14], [15]. Also, similar features of non-linear envelope response were recently shown in simulations for FNAL booster 
lattice [16]. An important feature of coherent non-linear resonant response is significantly different beam behavior depending on whether the resonance is crossed in the direction of increasing or decreasing space-charge effect (see Appendix A). Recently, an experimental study of this effect was performed by Uesugi et al. [18].

\section{HALF-INTEGER STRUCTURE RESONANCE}

One of the recently proposed SNS working points $\left(\nu_{0 x}, \nu_{0 y}\right)=(6.23,6.20)$ seems to be a good operational region due to the absence of dangerous imperfection resonances [21]. However, this working point lies very close to the systematic half-integer structure resonance with harmonic $n=12$ due to the SNS superperiodicity of 4 . It is thus extremely important to understand intensity limitations for this working point. This resulted in our studies of this working point with regard to the coherent-resonance condition and its applicability to the structure resonances.

\section{A. Uniform distribution}

We start our PIC simulations with a uniform-density KV beam. The maximum spacecharge tune shift of a full-intensity SNS beam with $N=2 \cdot 10^{14}$ protons can be as big as $\Delta \nu_{s c}=0.2$, depending on the beam distribution. For unbunched beam the effective number of particles should be increased to keep a similar equivalent space-charge tune shift. We use such an effective intensity parameter $\tilde{N}$ through our presentation of unbunched beams while realistic number of particles $N$ will be used for later description of bunched beams. Here we assume typical SNS beam parameters at energy $1 \mathrm{GeV}$ with unnormalized horizontal and vertical emittance $\epsilon_{x}, \epsilon_{y}=120 \pi \mathrm{mm} \operatorname{mrad}\left(\right.$ for a $\mathrm{KV}$ beam $\epsilon_{x, y}=4 \epsilon_{x, y, r m s}$ ). As a result, $\tilde{N}=6.6$ depresses betatron tunes towards the $2 \nu_{y}=12$ resonance. Note that no magnet errors are included in the simulations so that only lattice harmonics are present, with $n=12$ being the structure harmonic due the SNS superperiodicity of 4. Slightly higher intensity of $\tilde{N}=7.3$ distributes incoherent tunes around the $2 \nu_{y}=12$ resonance line which would 
ensure beam blow-up and particle loss following the standard single-particle approach. The corresponding tune foot-prints are shown in Fig. 5. However, for both intensities no $2 \nu_{y}=12$ resonance is observed as can be seen in Fig. 6, where rms beam emittances over the first 50 turns are plotted. The small emittance exchange observed in Fig. 6 is due to the coupled-tune working point with some details addressed in Ref. [19]. Following the coherent resonance condition in Eq. 1 our intensity should be increased by a factor $4 / 3$ to be at the middle of a coherent resonance $12=\Omega_{2}$. The corresponding tune distribution for $\tilde{N}=9.7$ is shown in Fig. 5. The tune foot-print is shown just after one turn but one can already notice the spread due to a resonance in the vertical direction. The associated increase of the vertical rms emittance is shown in Fig. 7. Due to the fact that the beam size was increased the effect of space charge was reduced as can be seen in Fig. 8 from the tune foot-print of particles after the increase of emittance saturated at 100 turns.

\section{B. Non-uniform distributions}

Once again, for non-uniform distributions the maximum space-charge tune shift is bigger than the one of a uniform beam. As a result, the difference between a single-particle approach and the coherent resonance condition becomes even more pronounced. Similar to the case of an imperfection resonance, we perform PIC simulations for various beam intensities to generate a resonance-response diagram for a WB distribution. Our major goal is to determine the onset of significant excursions in the beam-envelope response curve, and thus understand whether there is any intensity gain due to the coherent resonance condition in the vicinity of the structure resonance. Figure 9 shows the response diagram for this structure resonance. Perhaps unexpectedly, the noticeable excursions in the the response curve are comparable to the one driven by the imperfection errors in Fig. 4 . With intensities already higher than the incoherent space-charge limit, only a modest beating of the envelopes is observed. Clarification of such behavior in the vicinity of a structure resonance is given in Appendix B. 


\section{Bunched beams and multi-turn injection}

For a coasting beam, there is general agreement that the coherent-resonance theory applies and that the resonance condition is defined by the coherent frequencies. However, several effects should be taken into account (including the effect of images) when one wants to apply it to experimental observations. For bunched beams there is no good conceptual analytic framework. For long ellipsoidal bunches the transverse modes are decoupled from the longitudinal one but it is not clear to what extent the synchrotron motion will impact the resonance condition of the transverse coherent modes. In some accelerators where synchrotron motion is negligible (in the SNS the full injection process of 1000 turns takes about one synchrotron oscillation) it seems reasonable to expect that the impact of synchrotron motion will not be important. In fact, some recent experiments and simulations for the bunched beam in the LANL Proton Storage Ring (PSR) seem to support the above discussion [13] - [15]. Here we explore these effects for a realistic beam of the SNS.

Simulations are performed with 1052-turn injection for a beam with momentum spread of $d p / p=0.7 \%$. The tune foot-prints of a final full intensity beam are plotted at the end of accumulation process. Figure 11 shows foot-prints for three beam intensities $N=2 \cdot 10^{14}$ (red color), $N=3 \cdot 10^{14}$ (pink color), $N=4 \cdot 10^{14}$ (green color). Here $N$ is the number of protons in the SNS beam. Note that a $d p / p$ spread was present in the simulation but its effect on the tune spread was excluded from Fig. 11 for a clear presentation of the space-charge detuning discussed in previous sections. Modification of the space-charge induced foot-print by $d p / p$ spread is shown, as an example, for $N=2 \cdot 10^{14}$ in Fig. 12 and is discussed elsewhere [21], [24]. Time evolution of the vertical rms emittances corresponding to Fig. 11 is shown in Fig. 13. As expected, no effect of the resonance is observed until the beam gets into the bandwidth (response curve) of the coherent resonance which occurs around $N \approx 3 \cdot 10^{14}$ (Note that this does not mean that no particle loss is expected until $N \approx 3 \cdot 10^{14}$ because here only an effect of the $n / 2=6$ systematic resonance is considered with all magnet errors excluded from simulations). 


\section{ENVELOPE INSTABILITY}

In this section we extend the discussion on the half-integer resonance with space charge to include the issue of envelope instability, which was introduced in Ref. [29] for high current transport systems. To clarify the difference, we point out that the half-integer resonance is an integer resonance of the envelope $\left(n=\Omega_{2}\right)$, whereas the envelope instability is a halfinteger resonance of the perturbed envelope with the matched envelope of a periodic focusing system $\left(n / 2=\Omega_{2}\right)$; the latter is therefore an instability with exponential growth from an initial perturbation (likewise there exists an exponentially damped mode), while the former is independent from the initial mismatch. Since the matched envelope adopts the periodicity of the lattice this resonant instability occurs if the zero-current phase advance per focusing cell is above a quarter-integer, i.e. for $\sigma_{0}>90^{\circ}$. Space charge then leads to an extended stopband starting slightly below $\sigma=90^{\circ}$ as was discussed in detail in Ref. [29]. In the absence of space charge the envelope instability vanishes completely, in contrast with the half-integer resonance, which is shifted by space charge and also present without it.

In a circular machine lattice the envelope instability occurs if the basic focusing cell is at the same time a super period and $2 \nu / n$ approaches $1 / 2$. For a working point above $\nu_{0 x, y}=6$, as in the SNS, this would be the case if the basic 24 cells were all identical. As an example, we have calculated, in Fig. 14, the instability stopband for a symmetric FODO cell with $\sigma_{0}=96^{0}$ corresponding to $\nu_{0 x, y}=6.4$. We have used the KVXYG [23] code, which matches KV-envelopes and determines the eigenvalues (growth factors) of envelope perturbations. It is noted that the pronounced instability stopband with a growth factor above unity (likewise a damped solution with "growth factor" below unity) starts for full-current phase advance $\sigma<88.84^{0}$ (corresponding to a tune $\nu_{x, y}=5.92$ ). The strong flutter of the matched FODO envelope couples the in-phase and out-of-phase eigenmodes and leads to a single stopband.

The question may be raised if an envelope instability is also driven by an imperfection term as opposed to the strong "structure resonance" discussed above [28]. This might be expected, for example, in a lattice with working point above 6.25, if a 25-th harmonic 
gradient error were present $\left(n / 2=\Omega_{2}\right)$. We have taken a constant focusing lattice with $\nu_{0 x, y}=6.333$ and such a gradient error (symmetric), which implies $\sigma_{0}=91.2^{0}$ per error harmonic. For the quite large error of $4 \%$ a very narrow stopband of the out-of-phase eigenmode is found at $\sigma=89.59^{0}$, and a somewhat broader one of the in-phase mode at $\sigma=88.78^{0}$. Related to the full circumference the corresponding tunes are $\nu_{x, y}=6.222$ and $\nu_{x, y}=6.165$, which reflects the same coherent tune shift as found above for the half-integer resonance (Fig. 15). More important, we found that for errors of $2 \%$ and $1 \%$ the width of these stopbands decreases linearly with the error strength, hence the instability gets detuned at a very low level. A similar effect was found for a 1-st harmonic error, which can drive the same mode, but yields even more narrow stopbands. This allows the conclusion that the imperfection driven envelope instability for working points above the fractional tune of 0.25 (likewise 0.75 ) is ignorable. Note that the discussion above should not be confused with the 4th-order resonance driven by the octupole-like errors.

For completeness of the discussion we have also examined (with the KVXYG code [23]) the detailed picture of exact matching and appearance of an envelope instability (here of integer type $n=\Omega_{2}$ ) near the half-integer imperfection resonance which was considered in section III B (with symmetric error of $1 \cdot 10^{-3}$ ). The result is shown in Fig. 16, using on the abscissa the phase advance per period of the error (1/9-th of the circumference), hence the working point corresponds to $184^{0}$. The program determines the matched periodic envelope, which is plotted in black. Again, no effect is seen for the single particle tune passing through $180^{\circ}$. With increasing space charge, and the out-of-phase mode phase advance approaching $360^{\circ}\left(0^{0}\right.$ in the graph), the matched envelope develops an increasing flutter due to the gradient error (response curve of $n=\Omega_{2}$ resonance). The envelope beating, described as resonance curve in section III, (with initial envelope determined by ignoring the gradient error) must therefore be interpreted as oscillation about this new envelope matched to the error. The region where the out-of-phase mode phase advance is exactly $360^{\circ}$ is associated with a small stopband of the envelope instability (of the integer type $n=\Omega_{2}$, in contrast with the half-integer one discussed above). The relatively large width of this stopband can 
be understood as a result of the flutter of the matched envelope due to the vicinity of the half-integer imperfection resonance. There is, however, an uncertainty in the left boundary of this stopband since the program did not converge on a matched solution in the gap between $175.55^{0}$ and $174.1^{0}$.

In conclusion, we find that the suggestion of Ref. [27] to consider the envelope instability as a possible limitation to working points above quarter (or three quarter) integer fractional tunes should not be applied to the imperfection case of gradient errors. This has been confirmed by corresponding PIC simulations in the presence of gradient errors (no octupolelike errors) for a typical high-intensity ring tune depression, using the SNS lattice, which did not show any resonant behavior near a quarter integer fractional tune (or $n / 2=\Omega_{2}$ ).

\section{DISCUSSION}

An application of the coherent-resonance theory allows some increase in the space-charge limit due to the onset of the resonance at higher intensities than expected from a singleparticle approach (incoherent space-charge tune shift). The largest advantage occurs for the split-tune working point due to the difference between the coefficients $8 / 5$ vs. $4 / 3$ in the coherent space-charge limit condition, with $a=b$. For the split-tune case, an experiment was recently performed at LANL PSR [13]. A subsequent numerical simulations confirmed that observed beam broadening is due to the coherent half-integer resonance [14]. Also, both PIC simulation and direct solution of the envelope equations showed the non-linear response of the beam to this resonance [15]. Similar experiments were performed at other accelerators as well in an attempt to achieve higher beam intensity. For example, at the CERN Proton Synchrotron (PS), Cappi et al. [17] observed significant emittance growth at intensities higher than predicted by the space-charge limit based on the incoherent-tune approach. In fact, the data collected from various high-intensity machines [26], [27] show that the experimentally achieved space-charge tune shift is in good agreement with the coherent space-charge limit (Table I). 


\section{TABLES}

TABLE I. Comparison of experimentally achieved space-charge limit with coherent space-charge limit.

\begin{tabular}{|c|c|c|c|c|}
\hline & $\nu_{0 x} / \nu_{0 y}$ & $\Delta \nu_{i n c}$ & $\Delta \nu_{e x p}$ & $\Delta \nu_{c o h}$ \\
\hline KEK-B & $2.17 / 2.3$ & 0.17 & 0.23 & 0.27 \\
\hline FNAL-B & $6.7 / 6.8$ & 0.2 & 0.4 & 0.32 \\
\hline ISIS & $3.7 / 4.2$ & 0.25 & 0.4 & 0.4 \\
\hline AGS & $8.75 / 8.75$ & 0.25 & 0.58 & 0.33 \\
\hline AGS-B & $4.8 / 4.9$ & 0.3 & 0.5 & 0.48 \\
\hline CERN-PS & $6.22 / 6.22$ & 0.22 & 0.27 & 0.29 \\
\hline CERN-PS-2 & $6.22 / 6.28$ & $0.22 / 0.28$ & 0.36 & $0.35 / 0.37$ \\
\hline
\end{tabular}


In Table I, the incoherent space-charge limit $\Delta \nu_{i n c}$ is the distance from the bare tune to the $1 / 2$ resonance line, and $\Delta \nu_{\text {coh }}$ is the coherent space-charge limit calculated as $\Delta \nu_{i n c} / C_{2}$ (see Section II), with an approximate values for the $C_{2}$ coefficients in the rough assumption of $a=b$. To obtain more accurate numbers for the $C_{2}$ coefficients and corresponding space-charge limit one has to solve directly the dispersion relations in Ref. [12]. Note, that our calculated coherent space-charge limit is different from the one in [27] for the reasons summarized in Section V. Also, two limiting values are provided in the CERN-PS-2 example for the horizontal and vertical directions, respectively. It is clear, that the experimentally achieved $\Delta \nu_{\text {exp }}$ depends on many parameters, such as correction of the resonance line, limiting aperture of the machine, corresponding beam loss, etc. As a result, direct comparison of $\Delta \nu_{\text {exp }}$ with theoretically predicted $\Delta \nu_{c o h}$ should be done only for a specific machine knowing all the information hidden behind the "experimentally achieved" statement, as well as calculating more accurately $\Delta \nu_{c o h}$ for a specific beam parameters. Without taking into consideration these details we find a good agreement with the coherent space-charge limit: the largest envelope oscillations occur in the vicinity of the coherent limit resulting in beam loss and providing the experimentally achieved limit. Even for very limited-aperture machines it may be possible to approach the coherent space-charge limit by careful correction of the resonance line. For a machine with a large aperture to beam size ratio it may be, in fact, possible to slightly exceed the coherent resonant condition provided a good correction of the resonance strength is achieved (see Figs. 2 - 3). Once again, experimental data of Table I were adopted from Ref. [27] as a reference data for our comparison without entering into detailed discussion needed for specific machines. For an example of detailed study of this effect in a specific machine we refer to Refs. [13]- [15].

In this paper we have demonstrated the applicability of the coherent resonance spacecharge limit for the SNS case, including realistic beam parameters and the multi-turn injection process. Another finding is that no strong intensity limitation is expected in the vicinity of the resonance with a structure harmonic $n=12$ of the SNS lattice. Subsequent simulation in the presence of various magnet errors has shown that the main intensity limitation 
is expected due to the skew-quadrupole sum resonance. This prevents us from increasing the intensity significantly beyond $N=2 \cdot 10^{14}$ for the working point $\left(\nu_{0 x}, \nu_{0 y}\right)=(6.23,6.20)$ unless a correction scheme is applied or the working point is adjusted accordingly. Note that the coherent resonance condition is applicable to coupling resonances as well [12]. In fact, our studies of the skew-quadrupole sum resonance in the presence of space-charge suggests a beam envelope response similar to the one-dimensional resonance presented in this paper. These studies will be reported in a separate paper.

\section{SUMMARY}

In this paper we discuss the space-charge limit in the high-intensity rings based on the coherent resonance condition. The coherent resonance condition allows some increase in space-charge limit due to the onset of a resonance at higher intensities than expected based on the incoherent resonance condition. Application of this condition both to the imperfection and structure resonances are discussed. We also explore an applicability of such an effect to a realistic bunched beam and multi-turn injection process. In addition, we address the issue of the envelope instability in a circular machine.

\section{ACKNOWLEDGMENTS}

We are indebted to J. Holmes, R.L. Gluckstern and A. Burov for extensive useful discussions. We are also grateful to M. Blaskiewicz, Y.Y. Lee, Y. Papaphilippou, G. Parzen, J. Struckmeier and J. Wei for useful comments and discussions in the course of these studies.

\section{APPENDIX A: RESPONSE DIAGRAM}

The response curves in Figs. 2 - 4 show a typical non-linear response of an oscillator to a resonance. Both the phase-space diagrams and response curves can be obtained by applying the phase-amplitude averaging technique [30] and plotting solutions for the stationary fixed 
points. This technique was applied to the envelope equation in the vicinity of the half-integer resonance by Smith [4] and later systematically described by Sacherer [5]. We use Sacherer's notation for the fixed points to describe the response curves. Consider, for example, the blue curve in Fig. 3. A sudden jump in envelope excursion for a critical intensity parameter corresponds to the fact that for intensities lower than a critical value there is only one stable fixed point $S^{-}$while for intensities above critical value there are two stable fixed points $\left(S^{-}, S^{+}\right)$, with only $S^{+}$amplitudes plotted above the critical value in Fig. 3 . For the critical intensity, in the location of the jump, $S^{-}$corresponds to the upper point of the jump, $S^{+}$to the lower point, with $U^{+}$located in between. Here $U^{+}$stands for the unstable fixed point, $S^{-}$is the large-amplitude stable fixed point, and $S^{+}$is the small-amplitude stable fixed point. Configuration points near $S^{+}$and $S^{-}$oscillate with small amplitudes about these points whereas points near $U^{+}$may follow the separatrix and make much larger excursions.

A consequence of such response curves for situations with a slow adiabatic change in the intensity parameter $I$ is a different beam behavior depending on the direction in which the critical value is crossed. For the beam in the $S^{+}$state, if $I$ is decreased (for example due to the acceleration), the beam envelope jumps to the top of a response curve when a critical value is reached, which results in oscillations around $S^{-}$. As can be seen from Fig. 3, the maximum excursion corresponding to $S^{-}$decreases with further decrease of $I$. As a result, one can cross the resonance if the beam pipe aperture allows the maximum envelope excursion given by $S^{-}$. Clearly, the maximum of $S^{-}$decreases with the resonance correction as demonstrated in Fig. 3. In the other direction of increasing $I$, the beam envelope continues to grow along the $S^{-}$state which results eventually in a beam loss.

This effect of resonance crossing is demonstrated in Figs. 17 - 18 for the $10^{-3}$ error case of Fig. 3. We sweep over the stopband increasing space charge by parametrically increasing the perveance in the envelope equations (as might occur during rf capture) or, alternatively, in the opposite direction (during acceleration, for example). The resulting single-particle fractional tune is shown as well as the value of the fractional tune where the small amplitude envelope resonance with $n=9$ occurs (dotted line at $\nu_{y}=4.4375$, corresponding to $I=1.635$ 
in Fig. 3). In the first case the envelope oscillation maintains the condition of resonance far beyond the small amplitude resonance condition (near turn 60) by a steadily increasing amplitude; in the second case the envelope increase is limited as the system jumps across the resonance near the exact resonance condition (near turn 80) with a bounded envelope increase. For detailed mathematical description of this phenomena we refer the interested reader to [5], and for the experimental studies of this effect to [18].

\section{APPENDIX B: IMPERFECTION AND STRUCTURE RESONANCE}

As discussed in Section III, the width of beam response to a coherent resonance is a function of the amplitude of the introduced imperfection error. As a result, for a very large magnitude of an error a noticeable beam response (envelope beating) may start at intensities even lower than the incoherent space-charge limit. This, of course, can be determined by measuring the magnitude of the errors. Structure resonance may be regarded to as an imperfection resonance with a large magnitude of an error. How large the amplitudes of specific structure harmonics are depends on the specific lattice. One superperiod of the SNS lattice for the working point $\left(\nu_{0 x}, \nu_{0 y}\right)=(6.23,6.20)$ is shown in Fig. 10. Fourier analysis of the vertical beam envelope showed that the amplitude of harmonic $n=12$ is comparable in size to the amplitude of an error harmonic used in Section III, which explains a modest width of beam response curve near the structure resonance with this harmonic. For comparison, in the horizontal plane, Fig. 10 shows two strong peaks in each superperiod. Thus, the large amplitudes of structure harmonics at $n=4$ and $n=8$ are expected. Fourier analysis of the horizontal envelopes shows that the magnitude of these structure harmonics is approximately a factor of five stronger than the amplitudes of other nearby structure harmonics. As a result, for the SNS lattice, the coherent resonance with harmonic $n=12$ is not expected to introduce a significant intensity limitation which is in agreement with our PIC simulations shown in Fig. 9.

To summarize, the structure half-integer resonances are potentially very dangerous, and, 
if possible, the working point in the vicinity of such resonances should be avoided. It appears, however, possible to have a working point in the vicinity of a structure resonance without significant intensity limitation if the relative harmonic in the lattice is sufficiently weak. 


\section{REFERENCES}

[1] J. Wei, D.T. Abell, J. Beebe-Wang, M. Blaskiewicz, P. Cameron, N. Catalan-Lasheras, G. Danby, A.V. Fedotov, C. Gardner, J. Jackson, Y.Y. Lee, H. Ludewig, N. Malitsky, W. Meng, Y. Papaphilippou, D. Raparia, N. Tsoupas, W.T. Weng, R.L. Witkover, and S.Y. Zhang, Phys. Rev. Special Topics Accel. and Beams, V. 3, 080101 (2000).

[2] D.C. Morin, MURA Report 649 (Ph.D. thesis, University of Wisconsin, 1962).

[3] P. Lapostolle, Conf. on High Energy Acc. (Dubna, Russia, 1963), p. 1235.

[4] L. Smith, Conf. on High Energy Acc. (Dubna, Russia, 1963), p. 1232.

[5] F. Sacherer, Lawrence Rad. Lab Report UCRL-18454 (Ph.D. thesis, University of California, 1968).

[6] R.L. Gluckstern, Proceedings of LINAC’70 (Fermilab, Batavia, 1970), p. 811.

[7] I. Hofmann and K. Beckert, IEEE Trans. Nucl. Sci. NS-32 (PAC'85), p. 2264.

[8] S. Machida, Nucl. Inst. Meth. A309 (1991), p. 43; AIP Conf. Proceedings 448, (New York, 1998), p. 73.

[9] R. Baartman, AIP Conf. Proceedings 448, (New York, 1998), p. 56.

[10] A.V. Fedotov, R.L. Gluckstern and M. Venturini, ICFA Workshop (Wisconsin, USA, 1999), p. 27.

[11] M. Venturini and R.L. Gluckstern, Phys. Rev. ST AB, V. 3, 034203 (2000).

[12] I. Hofmann, Phys. Rev. E 57, p. 4713 (1998).

[13] J.D. Galambos, S. Danilov, D. Jeon, J.A. Holmes, D.K. Olsen, F. Neri and M. Plum, Phys. Rev. Special Topics - AB 3, (2000) 034201.

[14] J. Holmes, A. Aleksandrov, J. Galambos, D. Olsen, F. Merrill and B. Macek, Proceedings of EPAC'00 (Vienna), p. 936 (2000). 
[15] J. Holmes, V. Danilov, J. Galambos, A.V. Fedotov and R.L. Gluckstern, "Resonant beam response in the PSR accumulator ring", Proceedings of Particle Accelerator Conference (Chicago, USA, 2001).

[16] M. D'yachkov, R. Baartman and F. W. Jones, "Multiturn simulations of coherent betatron resonance with space charge", Proceedings of Particle Accelerator Conference (Chicago, USA, 2001).

[17] R. Cappi, R. Garoby, S. Hancock, M. Martini, J.P. Riunand, Proceedings of PAC'93, p. 3570.

[18] T. Uesugi, S. Machida, Y. Mori, Proceedings of EPAC'00 (Austria, Vienna, 2000), p. 1333 ; also T. Uesugi, Ph.D. dissertation (2000).

[19] A.V. Fedotov, J. Holmes and R.L. Gluckstern, Phys. Rev. Special Topics Accel. and Beams, V. 4, 084202 (2001).

[20] J. Galambos, J. Holmes, D. Olsen, A. Luccio and J. Beebe-Wang, ORBIT's User's Manual, SNS/ORNL/AP Technical Note 011 (1999).

[21] A.V. Fedotov, I. Papaphilippou, J. Wei and J. Holmes, BNL/SNS Tech. Note No. 091 (March 2001).

[22] The utility of rms approach was first demonstrated by P. Lapostolle for stationary distributions (CERN/ISR/D1/70-36); R.L. Gluckstern later showed that the rms version of KV equations remain valid for beam with axial symmetry (1970 Linac Conference, unpublished); these results were extended by F. Sacherer to distributions with elliptical symmetry (IEEE Trans. Nucl. Sci. NS-18, p. 1105, 1971).

[23] J. Struckmeier and M. Reiser, Particle Accelerators, Vol. 14, p. 227-260 (1984).

[24] A.V. Fedotov, N. Malitsky, Y. Papaphilippou, J. Wei and J. Holmes, "Excitation of resonances due to the space charge and magnet errors in the SNS ring", Proceedings of 
Particle Accelerator Conference (Chicago, USA, 2001).

[25] A.V. Fedotov, N. Malitsky and J. Wei, SNS/BNL Tech. Note 086 (2001).

[26] W. Chou, In ICFA Beam Dynamics Newsletter, No. 20 (1999).

[27] A.V. Burov, G.W. Foster, V.D. Shiltsev, "Space-charge compensation in proton boosters", Proceedings of Particle Accelerator Conference (Chicago, USA, 2001).

[28] A.V. Burov (private communication).

[29] I. Hofmann, L.J. Laslett, L. Smith and I. Haber, Particle Accelerators, Vol. 13, p. 145-178 (1983).

[30] N.N. Bogoliubov and Y.A. Mitropolsky, Asymptotic Methods in the Theory of Nonlinear Oscillations (Gordon and Breach, New York, 1961). 


\section{FIGURES}

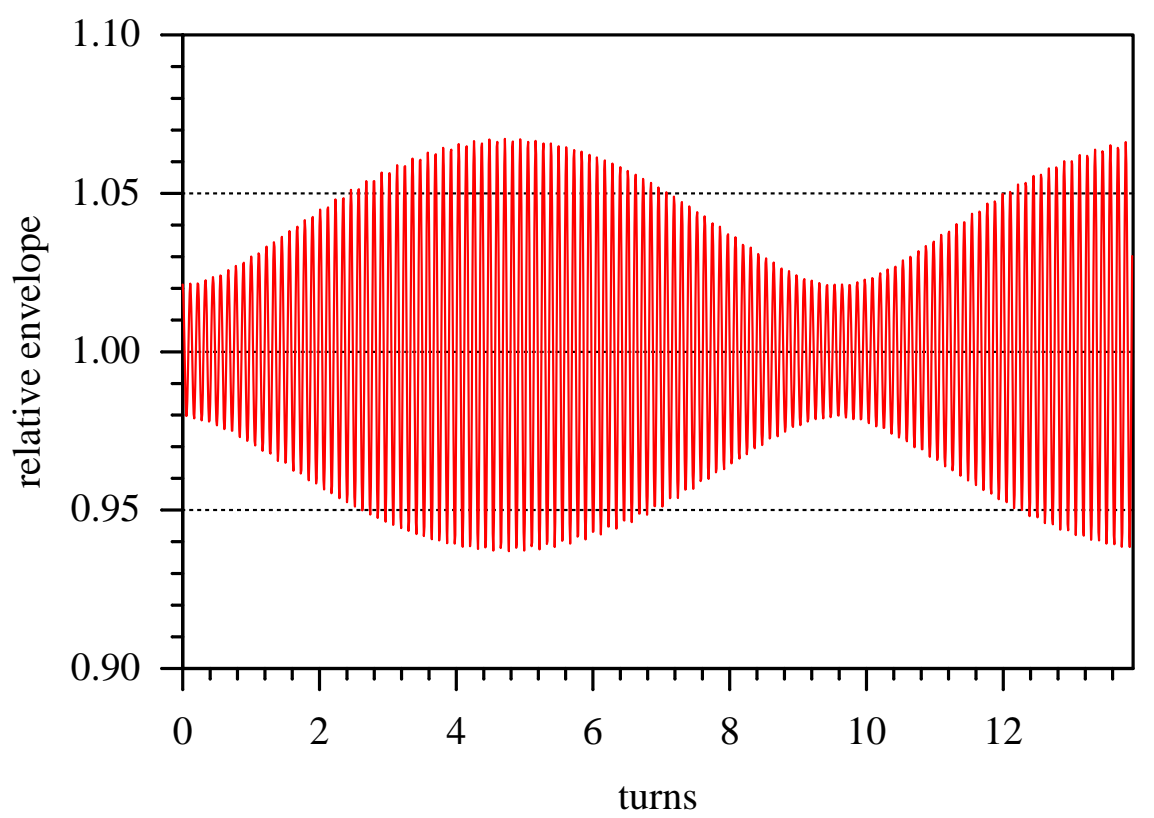

FIG. 1. Beating of envelope near half-integer imperfection resonance with $10^{-3}$ symmetric error at harmonic $n=9$ (single particle tune on resonance with $\nu_{x, y}=4.5$ ).

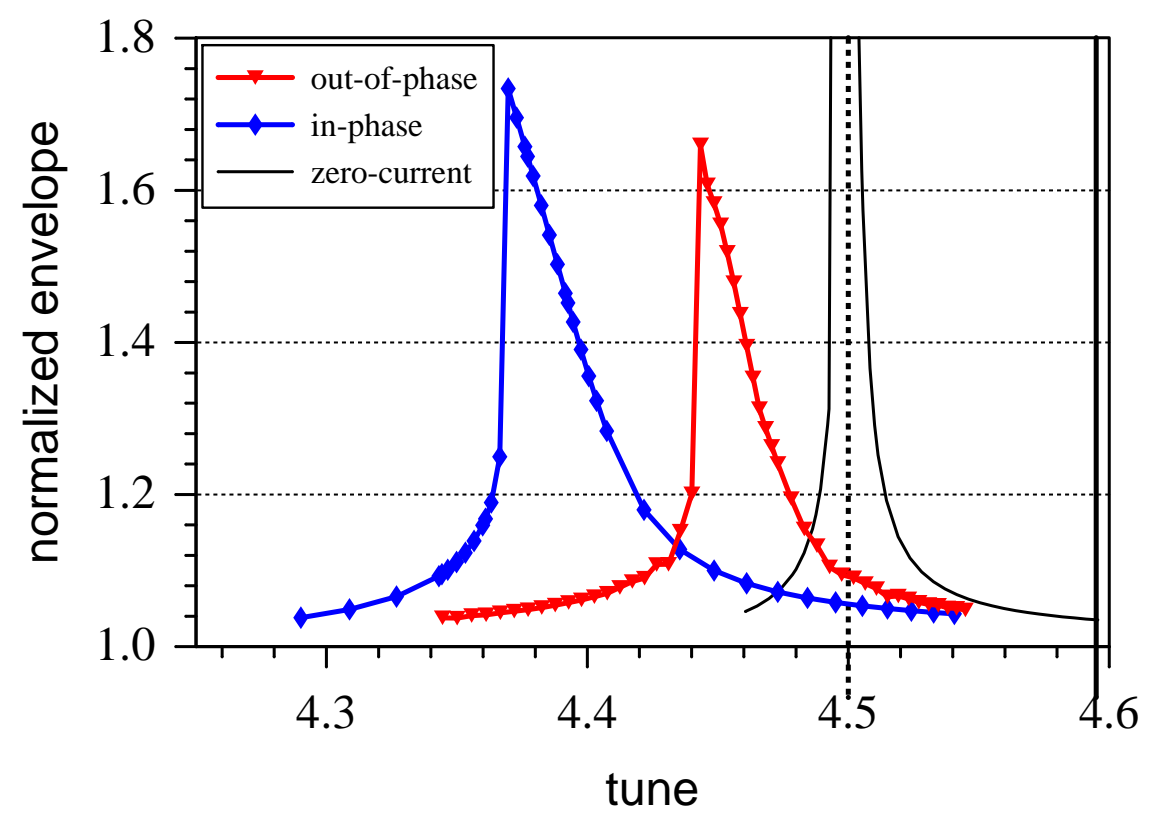

FIG. 2. Maximum envelope excursions (normalized to initial value) for symmetric and anti-symmetric focusing errors at variable intensity with fixed bare tune $\nu_{0, x, y}=4.6$, and for zero space charge case varying $\nu_{0, x, y}\left(10^{-3}\right.$ level error at harmonic $\left.n=9\right)$. 


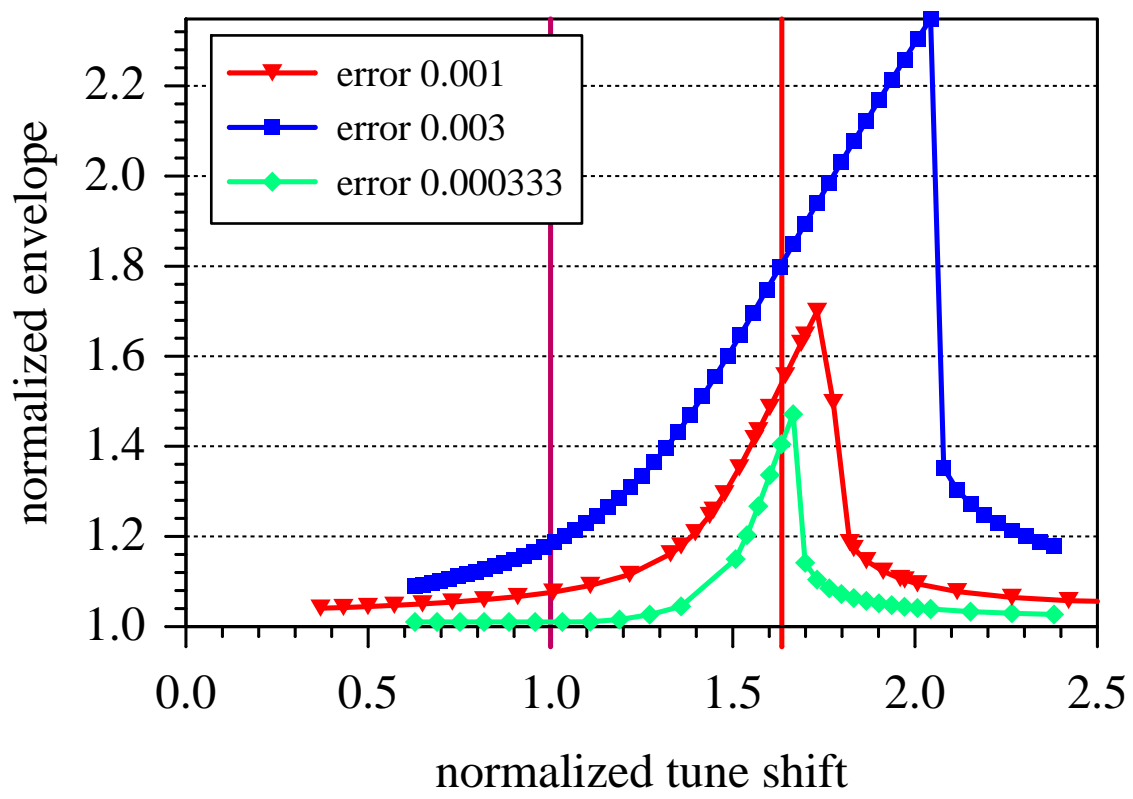

FIG. 3. Maximum $y$-envelopes excursions for three magnitudes of focusing errors with fixed $\left(\nu_{0 x}, \nu_{0 y}\right)=(6.45,4.6)$ as a function of normalized tune shift (intensity parameter $I$ with $I=1.635$ indicating the small-amplitude envelope resonance condition, and $I=1$ the single-particle resonance).

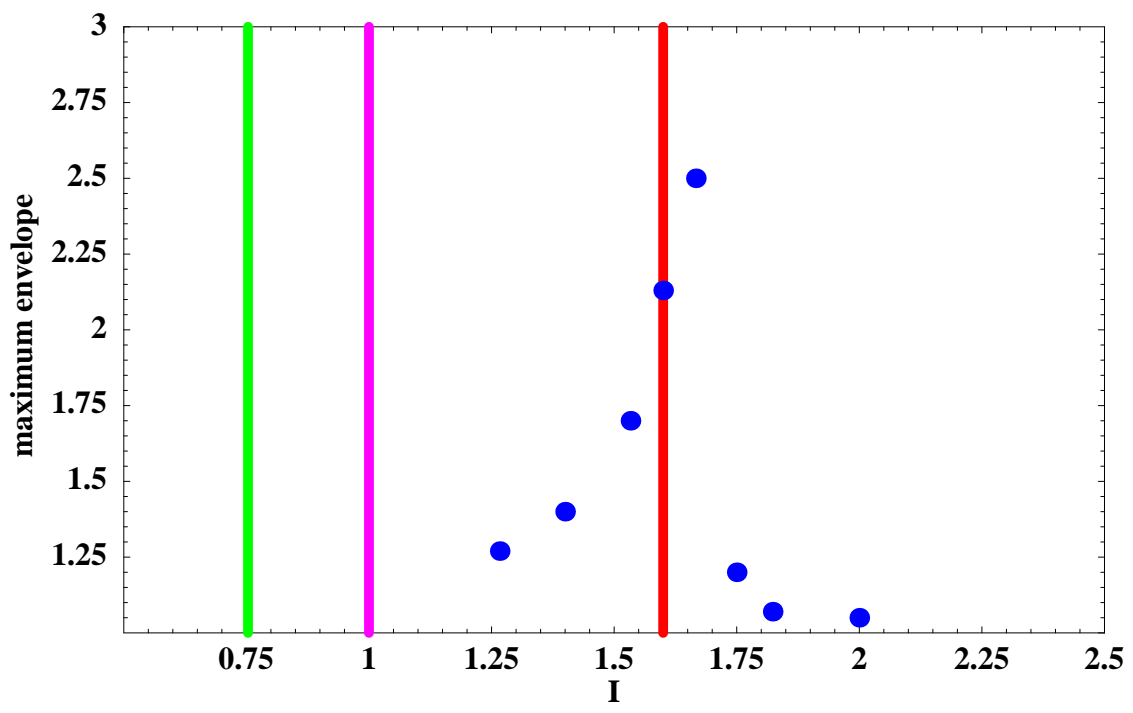

FIG. 4. Response of the vertical beam envelope to the coherent imperfection resonance at harmonic $n=9$. 


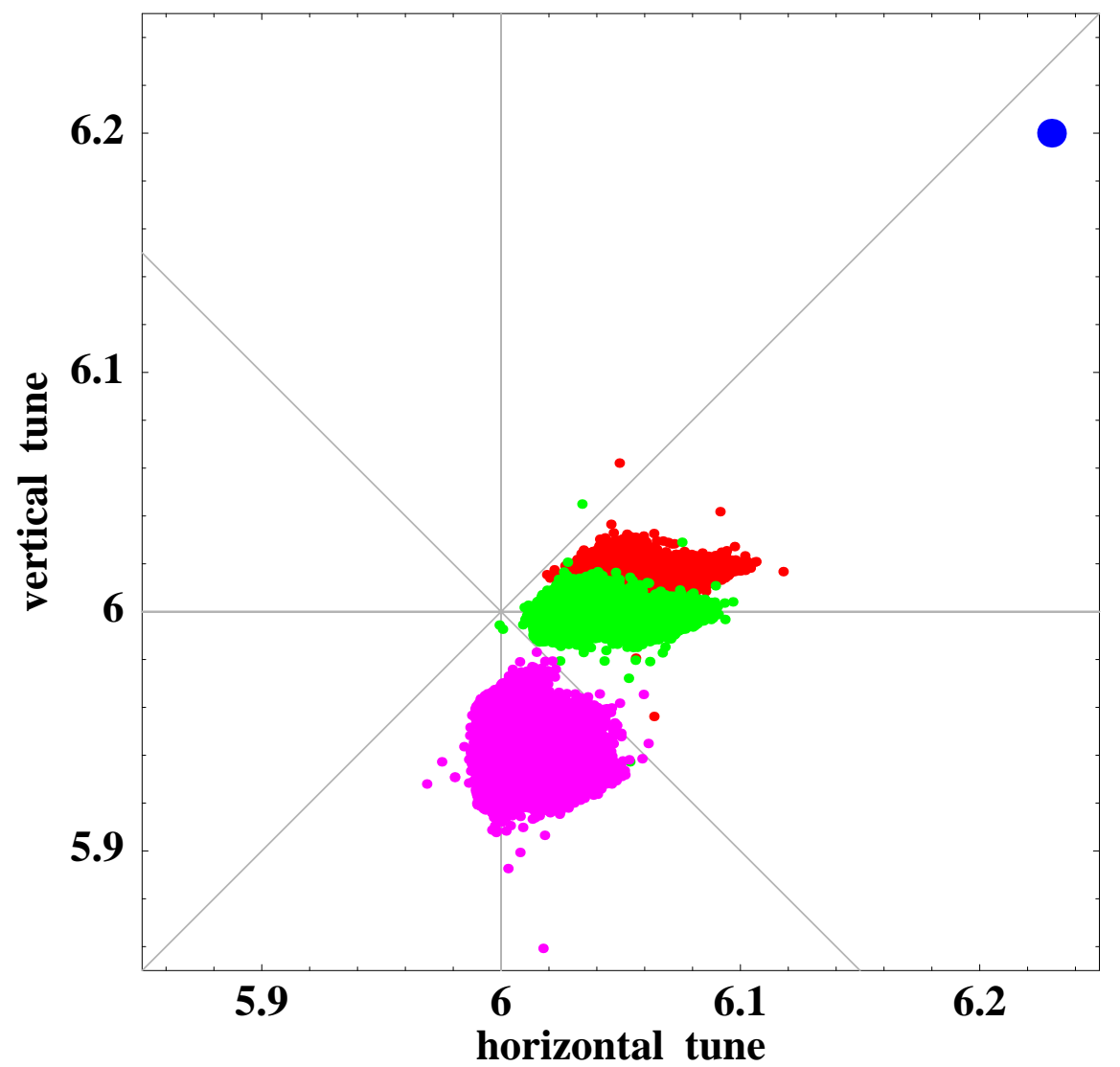

FIG. 5. Tune foot-prints of a KV beam for the SNS lattice working point $\left(\nu_{x}, \nu_{y}\right)=(6.23,6.20)$, shown by a blue dot. Intensities $\tilde{N}=6.6$ (red), $\tilde{N}=7.3$ (green), $\tilde{N}=9.7$ (pink).

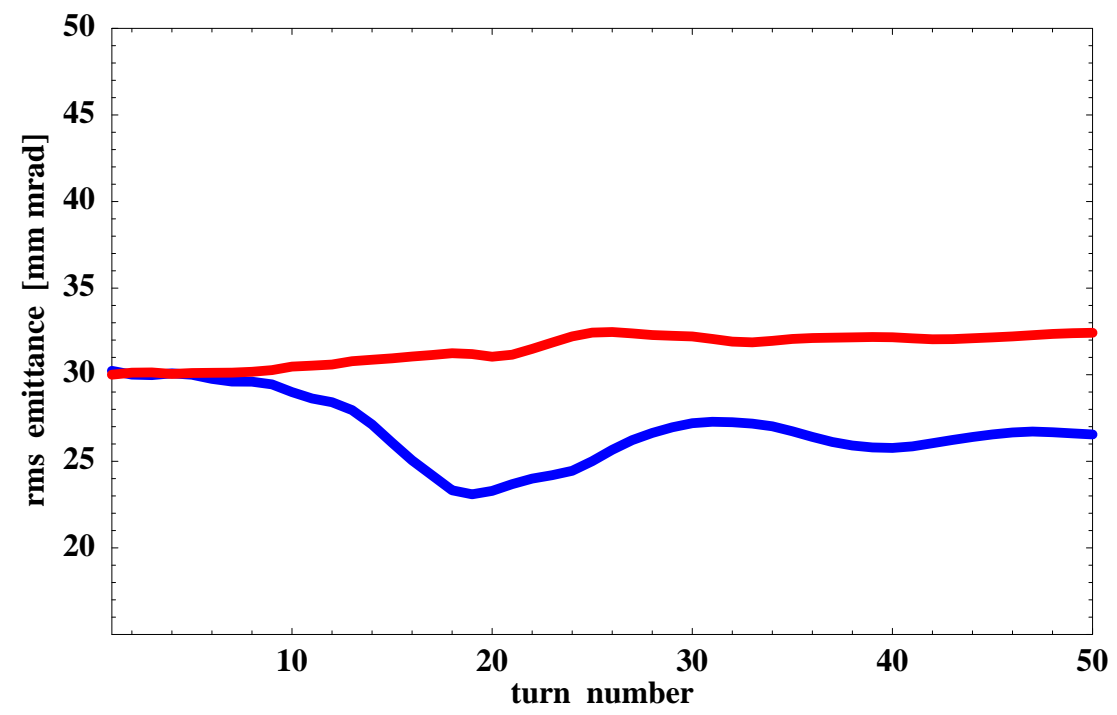

FIG. 6. RMS emittances of a $\mathrm{KV}$ beam for $\tilde{N}=7.3$. Vertical emittance is shown with red color while horizontal emittance is shown with blue color. 


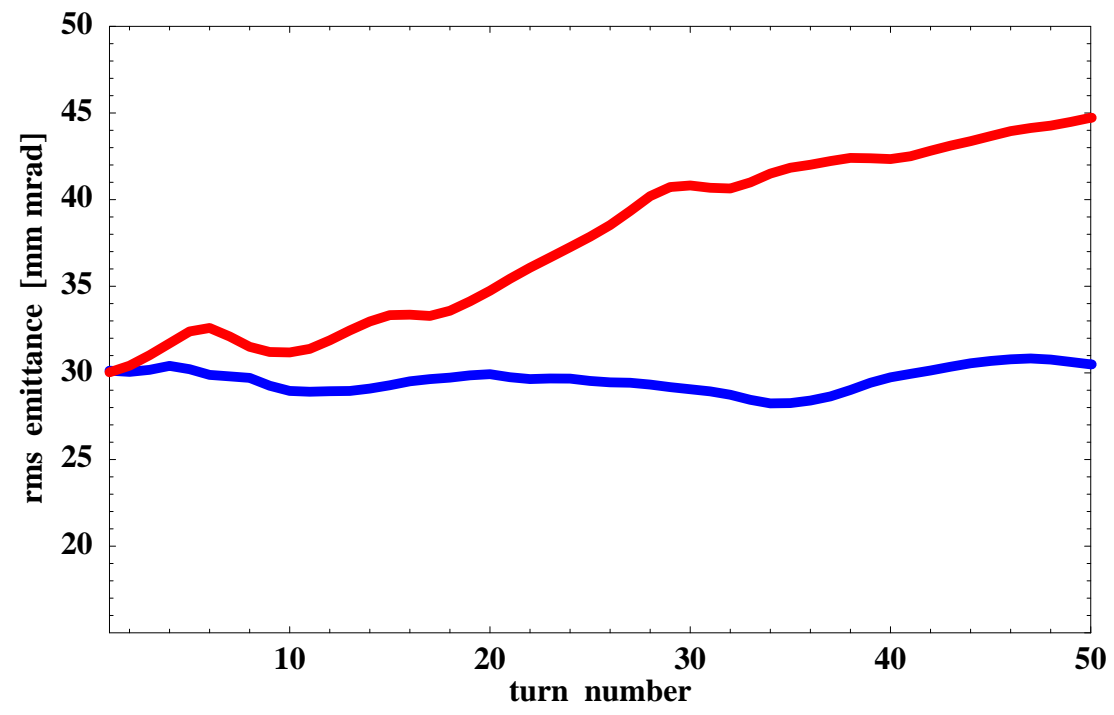

FIG. 7. RMS emittances of a KV beam for $\tilde{N}=9.7$. Increase of vertical emittance due to the $1 / 2$ coherent resonance.

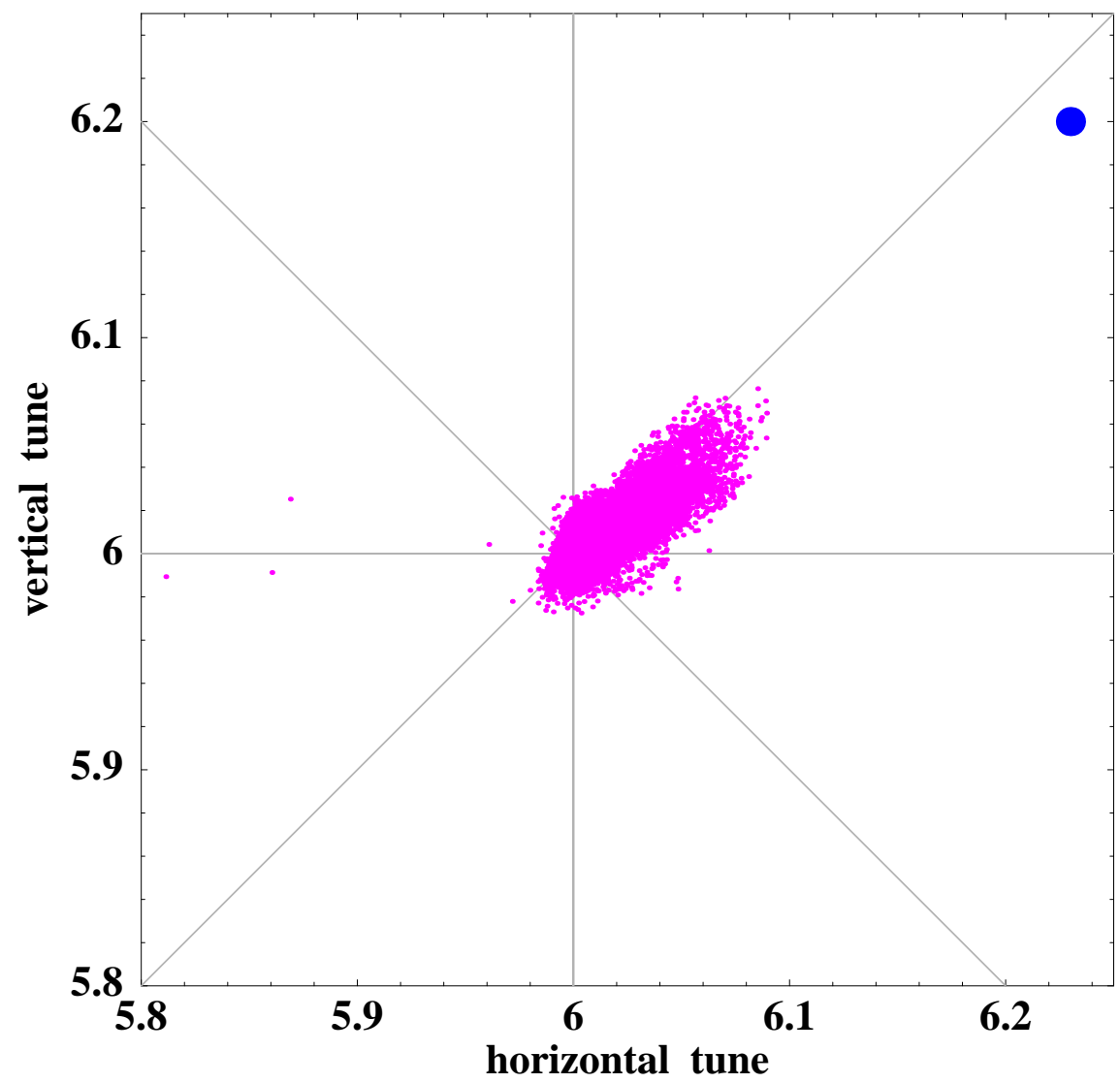

FIG. 8. Tune foot-print of a KV beam after saturated beam increase (at 100 turns) due to the coherent resonance for $\tilde{N}=9.7$. 


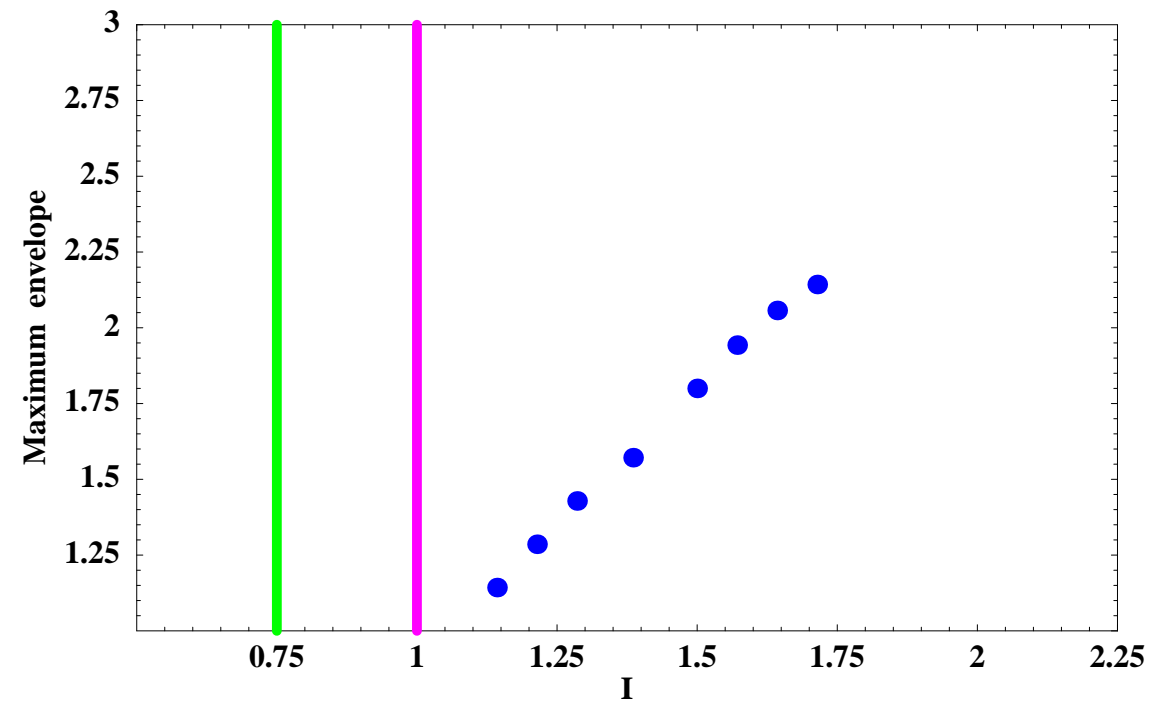

FIG. 9. Response of the vertical beam envelope to a coherent resonance with harmonic $n=12$.

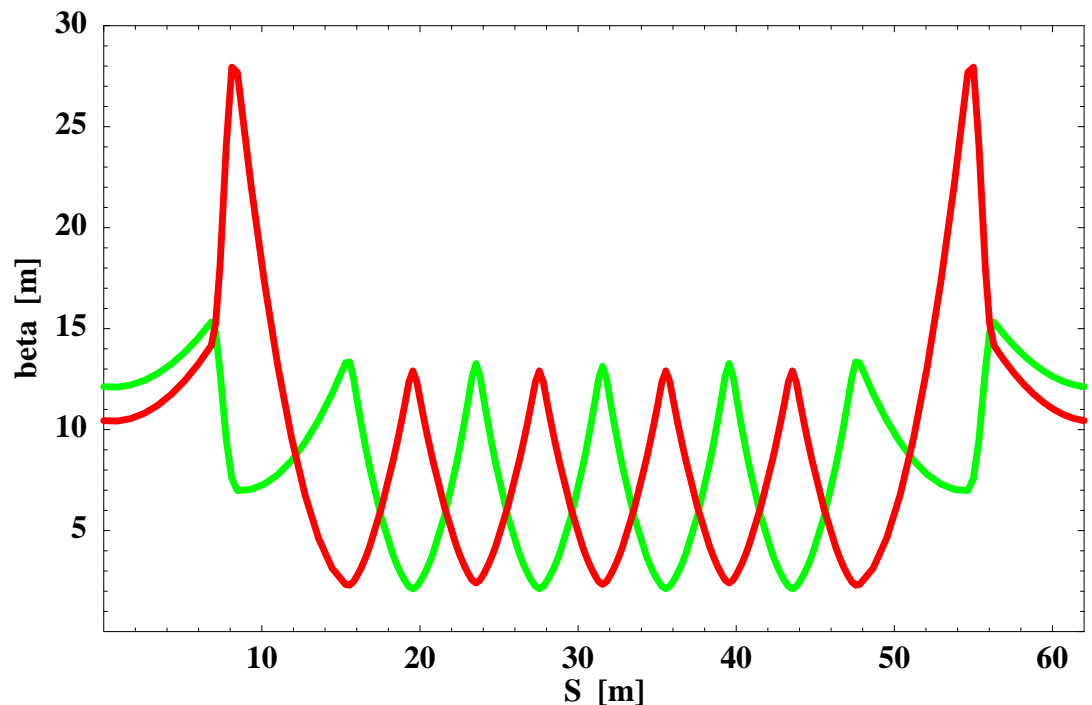

FIG. 10. Horizontal (red) and vertical (green) beta-functions of the SNS lattice with $\left(\nu_{0 x}, \nu_{0 y}\right)=(6.23,6.20)$ for one superperiod. 


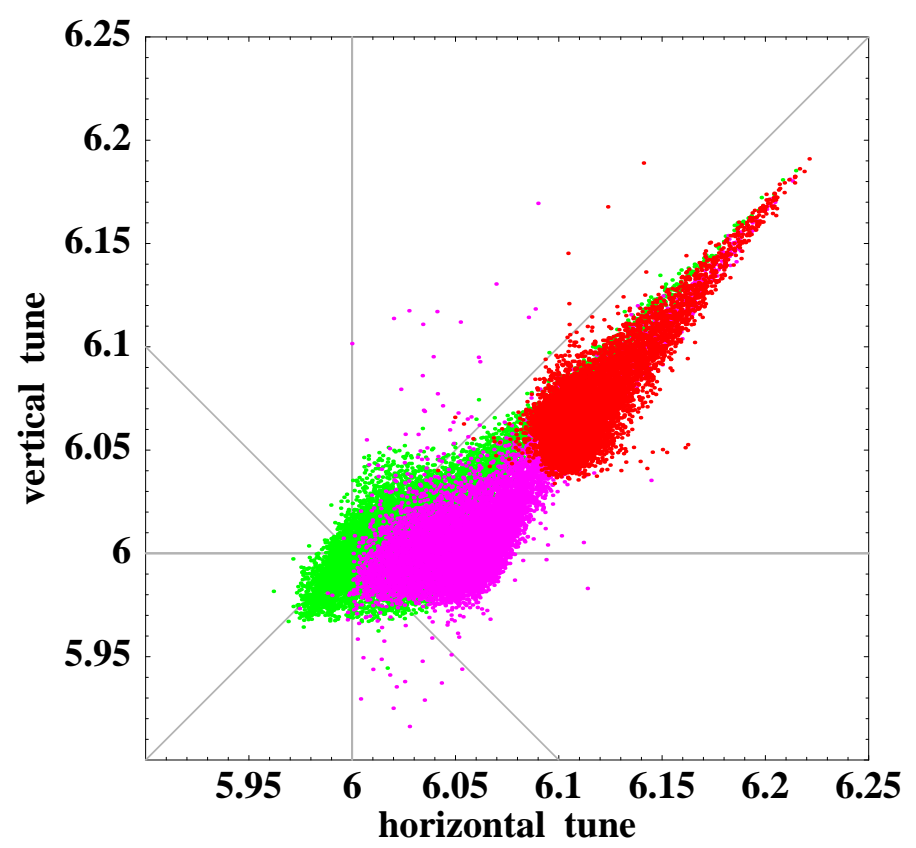

FIG. 11. Tune foot-prints at the end of 1052-turn injection process for the SNS beam. Intensities: $N=2 \cdot 10^{14}$ (red), $N=3 \cdot 10^{14}$ (pink), $N=4 \cdot 10^{14}$ (green) in the absence of magnet errors. SNS working point $\left(\nu_{0 x}, \nu_{0 y}\right)=(6.23,6.20)$.

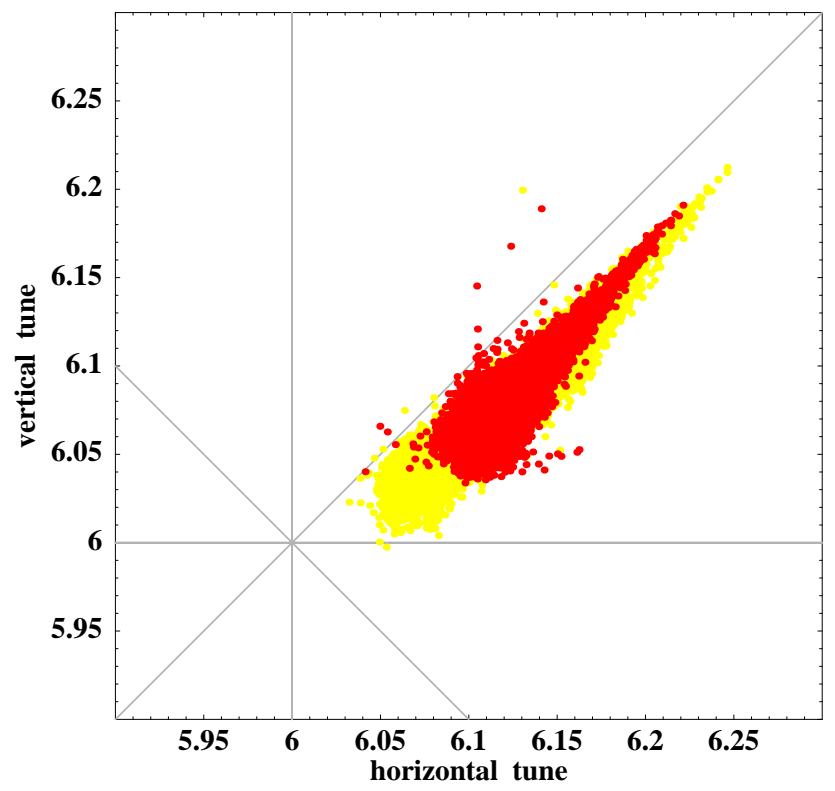

FIG. 12. Tune foot-print at the end of 1052-turn injection for the SNS beam with $N=2 \cdot 10^{14}$ particles. SNS working point $\left(\nu_{0 x}, \nu_{0 y}\right)=(6.23,6.20)$. Space-charge tune spread alone (red) and combined effect of the space-charge and chromatic detuning with $d p / p=0.7 \%$ (yellow). 


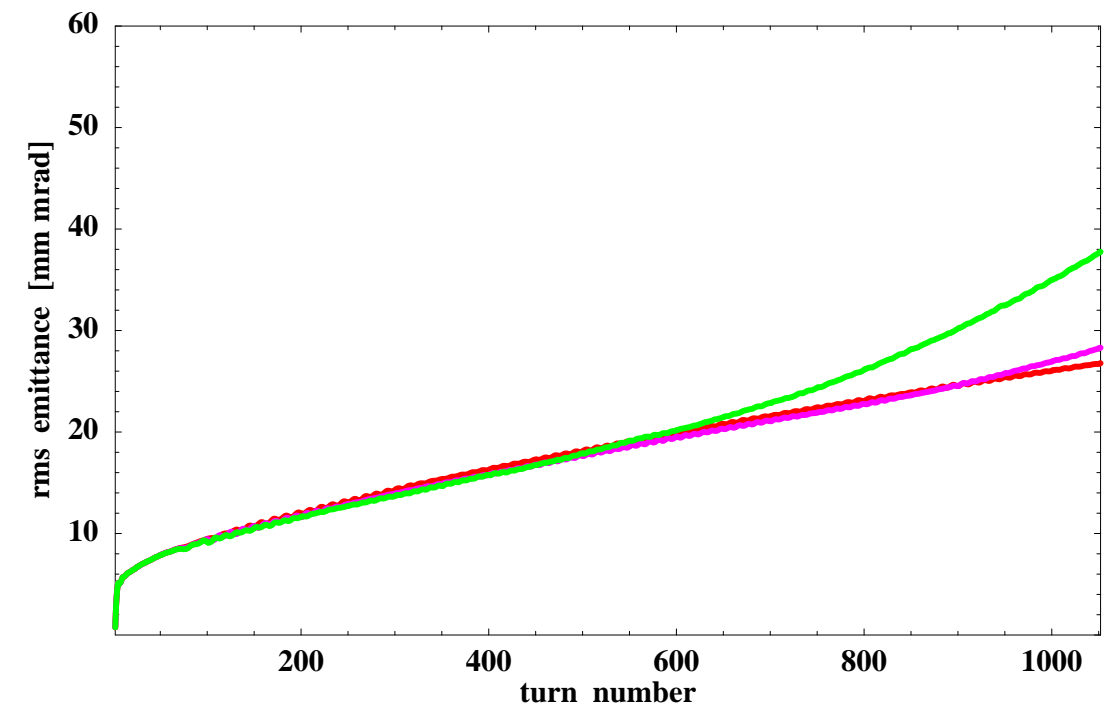

FIG. 13. Vertical rms emittances during the multi-turn injection process for three final intensities: $N=2 \cdot 10^{14}$ (red), $N=3 \cdot 10^{14}$ (pink), $N=4 \cdot 10^{14}$ (green), in the absence of magnet errors. SNS working point $\left(\nu_{0 x}, \nu_{0 y}\right)=(6.23,6.20)$.

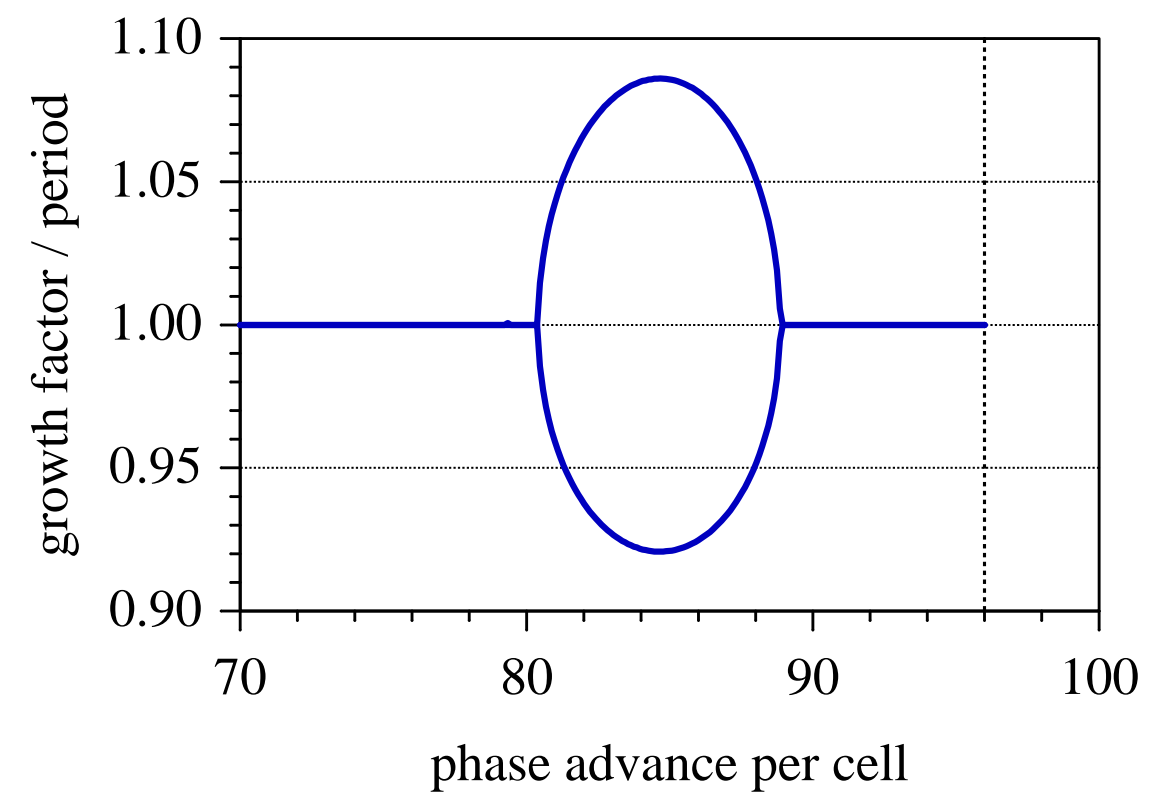

FIG. 14. Growth factors (per cell) for envelope instability of symmetric FODO cell with zero-current phase advance of $\sigma_{0}=96^{0}$, corresponding to $\nu_{0 x, y}=6.4$. 


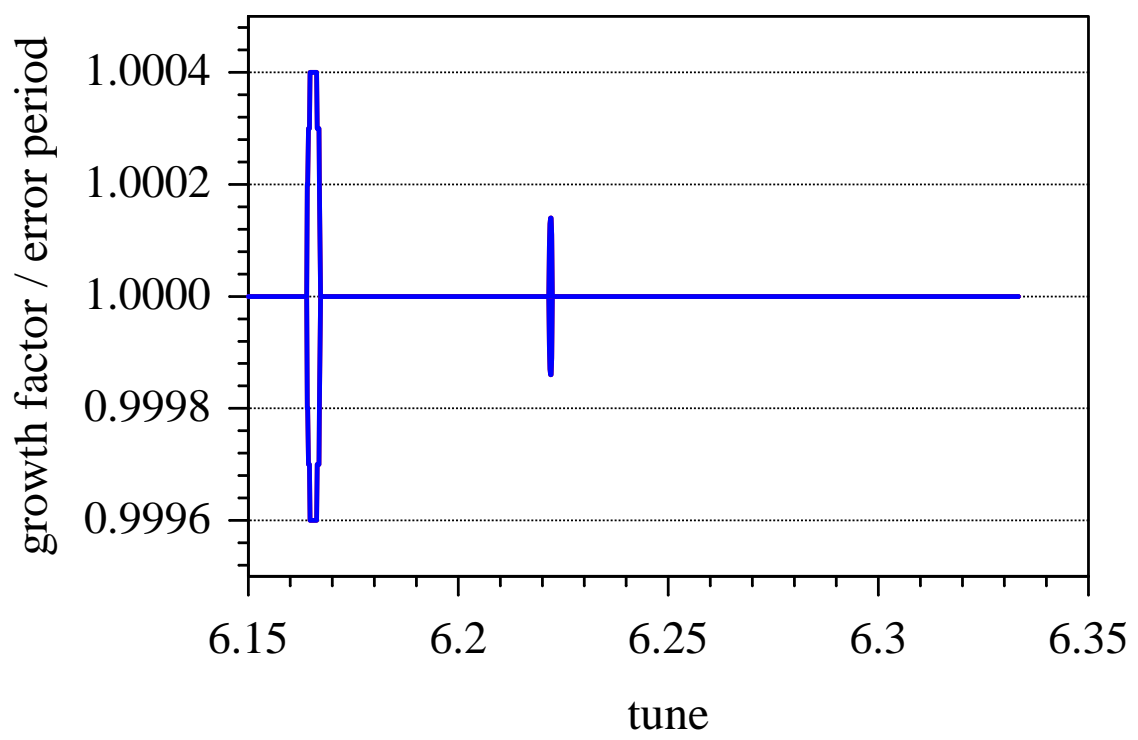

FIG. 15. Growth factors (per error period) for imperfection driven envelope instability with working point $\nu_{0 x, y}=6.333$ and error of $4 \%$ on harmonic $n=25$.

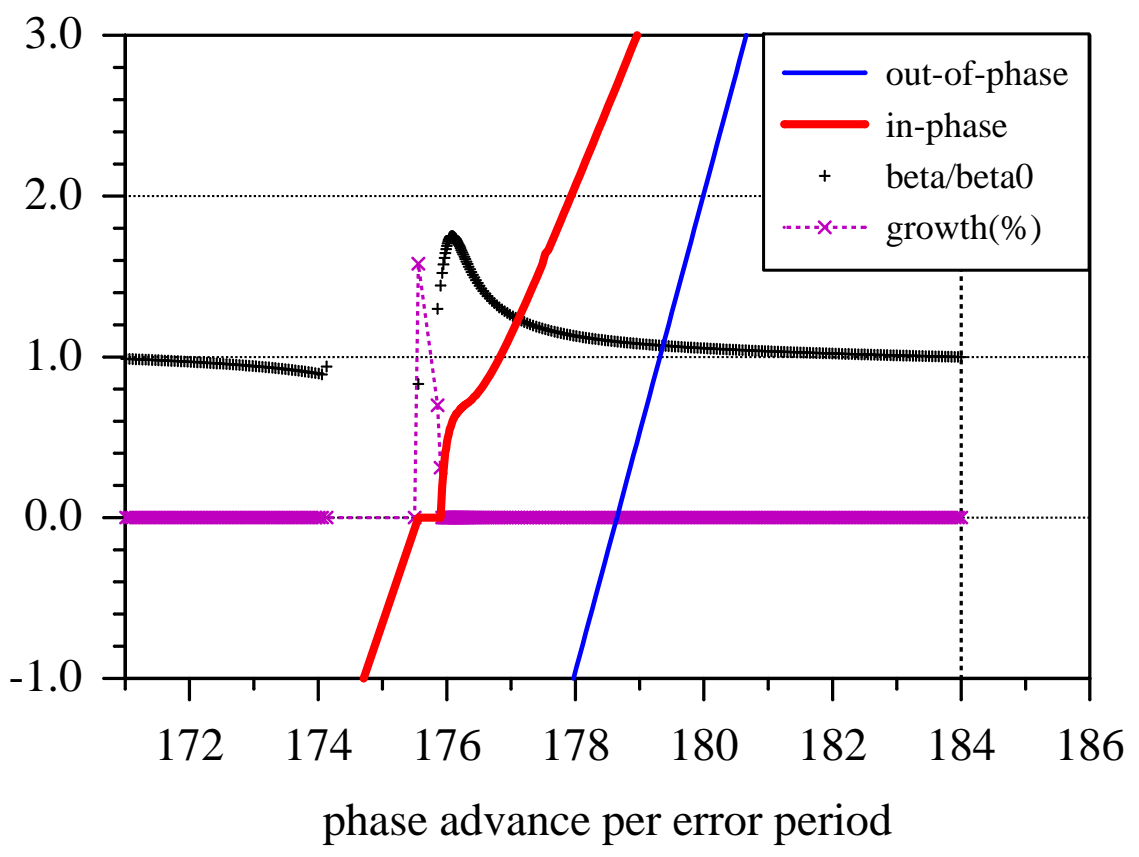

FIG. 16. Matched beta-functions (in units relative to zero space charge value), phase advance of envelope perturbations (in units of degrees deviating from $\left.360^{\circ}\right)$, and integer $\left(n=\Omega_{2}\right)$ envelope instability growth (in percent) for imperfection half-integer resonance as in Fig. 2. 


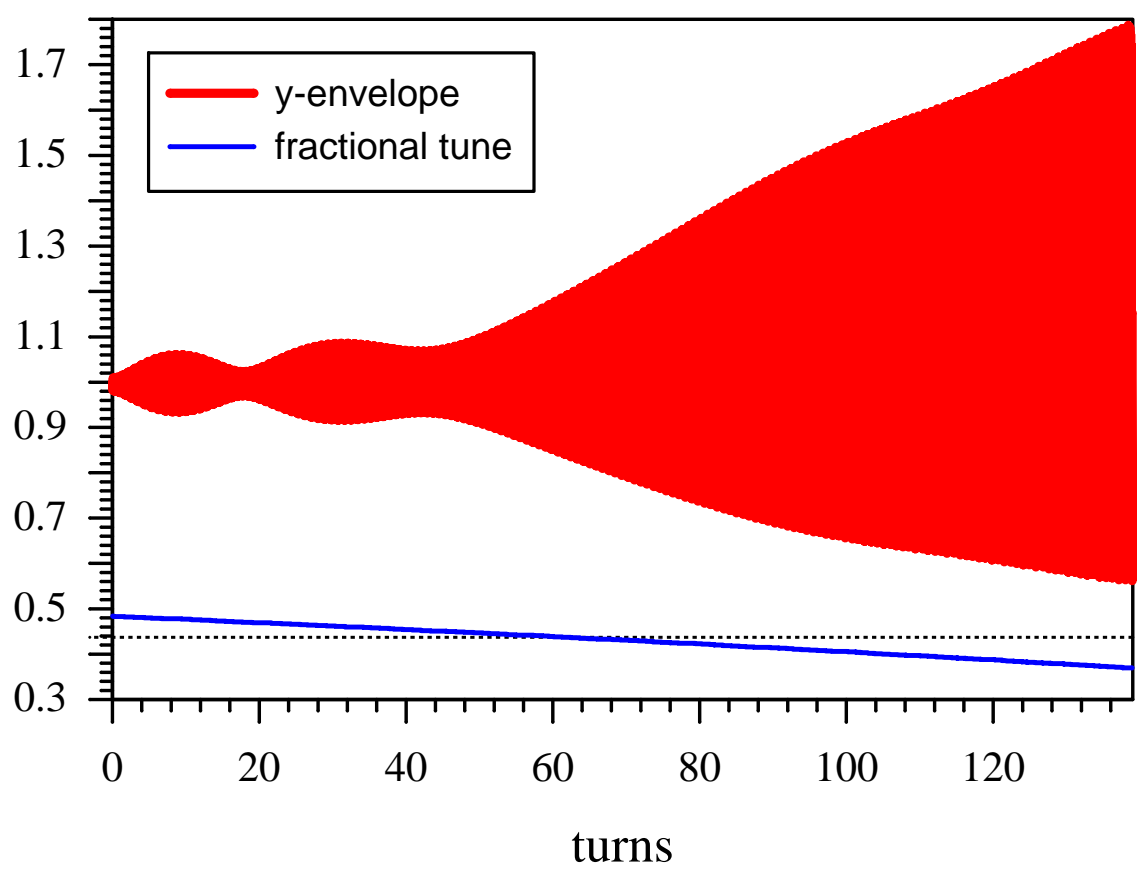

FIG. 17. Envelope for crossing over the stopband in the direction of increasing space charge ( $\nu_{y}$ decreasing linear in time from 4.48 to 4.37$)$.

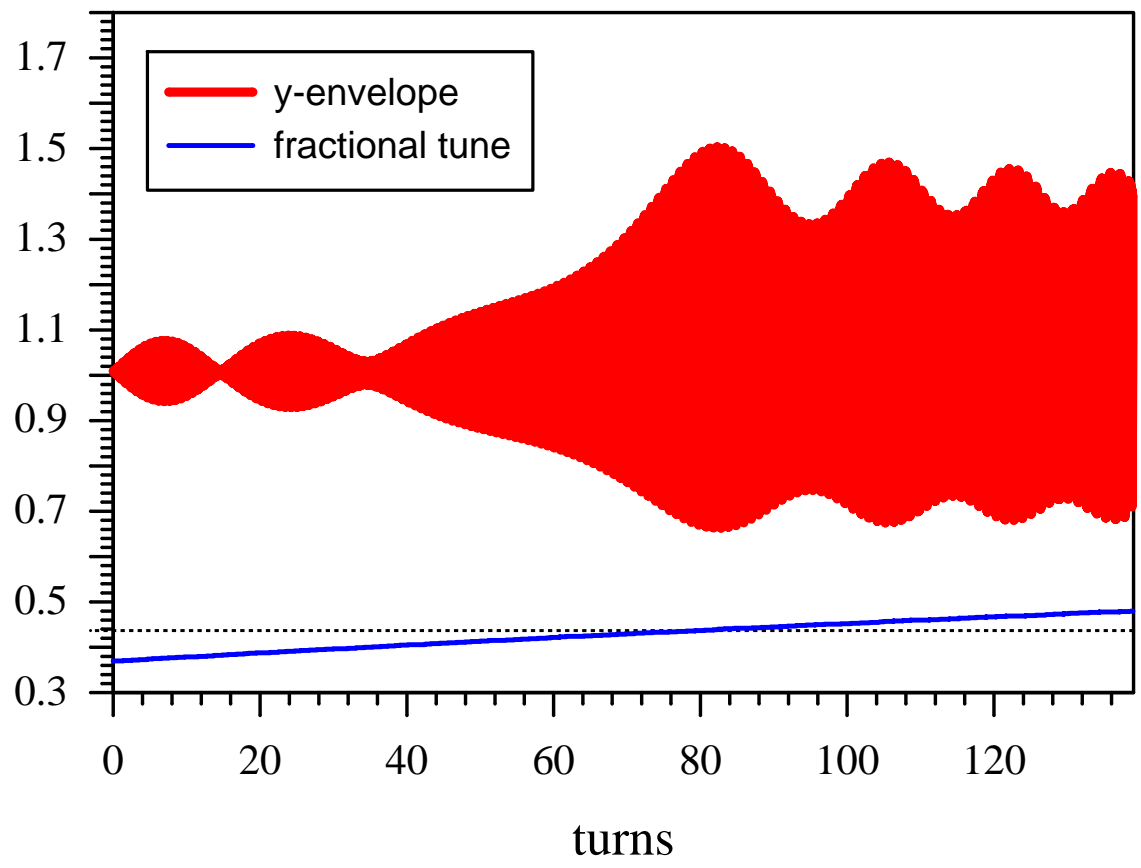

FIG. 18. Crossing of stopband with decreasing space charge $\left(\nu_{y}\right.$ increasing linear in time from 4.37 to 4.48$)$. 